\section{Micromelia, polysyndactyly, multiple malformations, and fragile bones in a stillborn child}

This is a brief report of a stillborn infant with a spectrum of distinctive congenital malformations. Although the family history and pregnancy gave no clue as to the aetiology of the malformations, we believe a description is warranted because the findings are so dramatic that, if further cases occur (or have occurred), they should be readily recognised and thus perhaps a syndrome could be delineated.

This $3026 \mathrm{~g}$ female infant was stillborn after 31 weeks' gestation to a 27 -year-old gravida 2 , para 1 mother. The parents were unrelated and an older female sib was healthy. Externally visible malformations included: an occipital encephalocele, frontonasal dysplasia with marked hypertelorism, microphthalmia, absence of the external nares, cleft palate, and micrognathia. The thorax was narrow and there was tetramicromelia and pre- and postaxial polysyndactyly of the hands and feet (fig 1).

Necropsy revealed absence of olfactory bulbs, hydrocephalus, pachygyria, and hypoplasia of the anterior and middle fossae. The lungs were hypoplastic, there was a

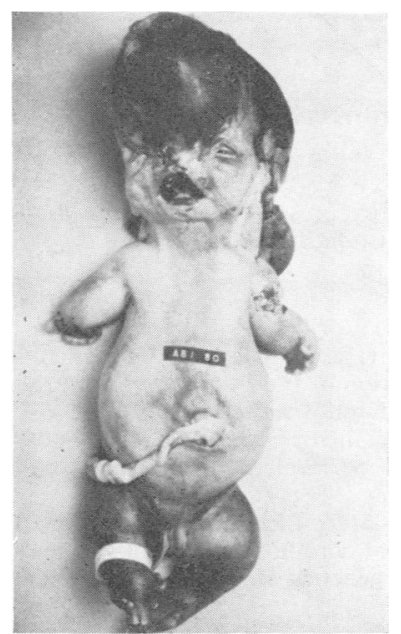

FIG 1 Post mortem photograph illustrating the majority of external features described in the text.

Received for publication 22 December 1981.

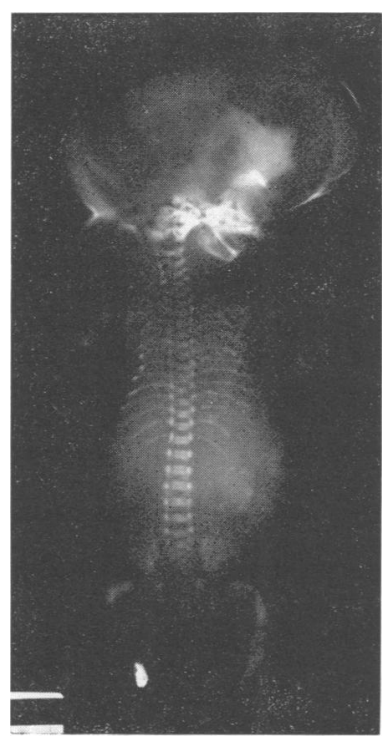

FIG 2 Radiograph showing widened sutures and thin gracile bones with fractures.

secundum type atrial septal defect, agenesis of the innominate vein, a persistent left vena cava draining into the coronary sinus, and a retroesophageal right subclavian artery. A hypoplastic gallbladder and the liver were midline and there was intestinal malrotation. There was bilateral cystic dysplasia of the kidneys with hydroureter and cystic dysplasia of the pancreas. The uterus was bifid, the cervix double, and the vagina single. A total body radiograph (fig 2 ) was of relatively poor quality but confirmed widened cranial sutures and multiple fractures of the long bones and the ribs, which were short and gracile. There were 11 pairs of ribs. The left clavicle was thick and the modelling of certain bones was ill-defined. Most notable was the right tibia which appeared double. A G banded karyotype from skin fibroblasts was normal.

\section{Blair F Carpenter and Alasdair G W Hunter Divisions of Pathology and Genetics, Eleanor Patterson Laboratory, Children's Hospital of Eastern Ontario, Ottawa, Ontario, Canada}

Requests for reprints to Dr A G W Hunter, Division of Genetics, Children's Hospital of Eastern Ontario, 401 Smyth Road, Ottawa, Ontario KIH 8LI, Canada. 